\title{
Sensitivity Enhancement of Ring Laser Gyroscope Using Dielectric-Graphene Photonic Crystal
}

\author{
Maryam Soltani, Alireza Keshavarz, Gholamreza Honarasa, and Reza Ghayoor \\ Department of Physics, Shiraz University of Technology, Shiraz, Iran \\ Corresponding author Email: m.soltani@sutech.ac.ir
}

Received: Dec. 9, 2017, Revised: Jan. 11, 2018, Accepted: Feb. 12, 2018, Available Online: June. 30, 2019

DOI: 10.29252 /ijop.13.1.53

\begin{abstract}
In a ring laser gyroscope, due to the rotation and the Sagnac effect, a phase difference between the two counter-propagating beams is generated. In this device, the higher phase difference between these two beams causes the better the interference pattern detection, and thus the sensitivity is increased. In this paper, the effect of inserting a dielectricgraphene photonic crystal inside a ring laser gyroscope on the interference pattern and the sensitivity of the device are studied and simulated using ABCD propagation matrix method. Results show that dielectric-graphene photonic crystal has a high transmission and therefore high efficiency in the wavelength of ring laser gyroscope. So it is a suitable choice to use in the ring laser gyroscope. Also, a comparison between ring laser gyroscope with and without dielectric-graphene photonic crystal shows that when the dielectric-graphene photonic crystal is in the system it is possible to build gyroscope with smaller lengths and high sensitivity.
\end{abstract}

KEYWORDS: ABCD propagation matrix method, Dielectric-graphene photonic crystal, Ring laser gyroscope, Sensitivity enhancement.

\section{I.INTRODUCTION}

The gyroscope is a key sensor in modern navigation systems enabling to plan, record and control the movement of a vehicle from one place to another. This device has a wide spectrum of applications in space engineering, aeronautical and military industry, automotive, medicine and so on[1]. There are several types of gyroscopes, including ring laser gyroscopes (RLG) [2]. Due to the importance of this device in various applications, especially military applications, extensive efforts have been made to increase the sensitivity of the device and improve its performance including using fast-light-enhanced $\mathrm{He}-\mathrm{Ne}$ ring-laser gyroscope [3], superluminal ring laser [4], multilayer optical coatings on one of the gyroscope mirrors[5], nonlinear effects [6] and so on. The maximum sensitivity measured in all of these methods was of the order of $10^{6}[7$, $8]$. The purpose of this paper is to increase the sensitivity of the device using photonic crystal.

Photonic crystals (PC) can be considered as periodic arrangements of regularly shaped materials with different dielectric constants. Periodicity can vary from single-dimensional (1D) to three-dimensional (3D) [9, 10]. The first research on photonic crystals refers to 1987[11]. Today, photonic crystals have many applications such as using photonic crystal for sensing applications [12, 13, 14], ultrafast switching [15], Highly nonlinear and highly birefringent dispersion compensating [16], linear and nonlinear photonic crystal waveguides $[17,18]$ and so on. The photonic crystal that is used in this paper is dielectricgraphene photonic crystal.

Graphene has high mobility and optical transparency and transmits light at visible wavelengths by $97.7 \%$ [19]. Therefore, entering a structure including graphene, in comparison with other structures, inside the laser gyroscope causes low absorption of the beam within the structure and increases the efficiency of the device.

In this paper, the effect of inserting a dielectric- graphene photonic crystal on the 
phase difference between two beams in a ring laser gyroscope is investigated using $\mathrm{ABCD}$ propagation matrix method and its effect on the interference pattern as well as the sensitivity of the device are simulated. Results show that the transmittance of dielectricgraphene photonic crystal in the wavelength of $\mathrm{He}-\mathrm{Ne}$ laser $(632.8 \mathrm{~nm})$ is approximately $80 \%$ and therefore the device has a good efficiency in this wavelength. A comparison between usual RLG and dielectric-graphene photonic crystal RLG shows that when the dielectricgraphene photonic crystal is in the system it is possible to build gyroscope with smaller dimensions and reduce the size of the gyroscope. Also, the use of this photonic crystal within a RLG increases the phase difference between two beams and the sensitivity of the device.

\section{THEORETICAL BASICS}

\section{A. Ring Laser Gyroscope}

A ring laser gyroscope consists of a triangular or square ring resonator. The gyroscope is based on the Sagnac effect. As a result of that, two counter-propagating waves traveling through the same closed path will exhibit a phase difference proportional to the angular velocity of the system. A schematic view of a triangular ring laser gyroscope is shown in Fig. 1. The laser source that is used in this device is $\mathrm{He}-\mathrm{Ne}$ laser and generates two counterpropagating beams. If the system is at rest, both light beams travel through the same optical path and stay in phase. If a rotation in a clockwise direction and angular velocity $\Omega$ is applied to the system, a difference in the optical path between the two beams will occur and can be calculated as follows [20]:

$\Delta L=c t_{+}-c t_{-}=2 \pi R\left(\frac{1}{1-\frac{\Omega R}{c}}-\frac{1}{1+\frac{\Omega R}{c}}\right) \approx \frac{4 \pi \Omega R^{2}}{c}(1)$

where $t_{+}$and $t_{-}$are the traveling times of the co-rotating and counter-rotating beams respectively, $R$ is the radius of the closed path and $c$ is the velocity of light in vacuum.
Therefore, the frequency difference between two beams is:

$\Delta \vartheta=\frac{4 A . \Omega}{\lambda L}$

where $A$ is the area of the closed path, $\lambda$ is the wavelength of the He-Ne laser $(632.8 \mathrm{~nm})$ and $L$ is the optical path. The phase difference between the two beams can be expressed as follows:

$\Delta \varphi=\frac{2 \pi \Delta \vartheta}{c} L=\frac{8 \pi \omega A}{c \lambda}$

The higher phase difference between these two beams causes the better interference pattern detection, and thus the sensitivity is increased. Here, we insert dielectric- graphene photonic crystal inside one of the ring laser gyroscope arms and simulate its effect on the interference fringes and sensitivity of the device by $A B C D$ propagation matrix method.

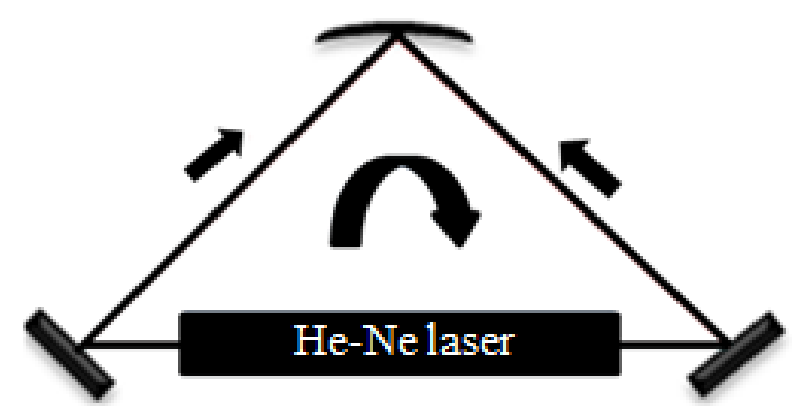

Fig. 1. A schematic view of a triangular ring laser gyroscope. The light is generated by the laser and transmitted through two outputs of the laser in two opposite directions.

\section{B. Dielectric-graphene photonic crystal}

Figure 2 shows the structure of a dielectricgraphene photonic crystal unit cell.

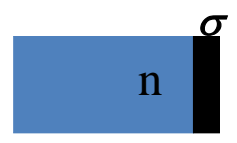

Fig. 2. The structure of the dielectric-graphene unit cell.

In this structure, the thickness of dielectric layer is $\mathrm{d}$ and the thin layer graphene has the conductivity, $\sigma$. The dielectric-graphene interface matrix that can link the field of the layer $n$ to the layer $n+1$ is [21]: 


$$
M=\left[\begin{array}{cc}
\left(2+i \kappa_{n} \xi\right) / 2 u_{n} & -i \kappa_{n} \xi / 2 \\
i \kappa_{n} \xi / 2 & \left(2-i \kappa_{n} \xi\right) u_{n} / 2
\end{array}\right]
$$

where parameters $\xi, \kappa_{n}$ and $u_{n}$ are defined as follows:

$\xi=\frac{\sigma \eta_{0}}{i k_{0}}$

$\kappa_{n}=\frac{k_{n}}{\varepsilon_{n}}$

$k_{n}=\sqrt{\varepsilon_{n} k_{0}^{2}-k_{z}^{2}}$

$k_{z}=k_{0} \sin \theta$

$u_{n}=\exp \left(i k_{n} d_{n}\right)$

where $\eta_{0}$ is air impedance, $k_{0}=\frac{2 \pi}{\lambda}, \theta$ is the angle of the incident beam to the structure, and the conductivity of graphene is obtained as:

$\sigma=\frac{i e^{2} \mu_{c}}{\pi \eta^{2}\left(\omega+i \tau^{-1}\right)}$

where $e$ is electron charge, $\mu_{c}$ is the chemical potential of graphene and $\tau$ is the relaxation time of charge carriers. If the unit cell structure of Fig. 2 repeats periodically for a number of periods, such as $N$, the entire matrix is considered as [22]:

$U=\prod_{n=1}^{N} M_{n}$

After obtaining the matrix $U$, transmission coefficient and the phase delay of the photonic crystal can respectively be obtained:

$t=\frac{1}{U(1,1)}$

$\phi=\tan ^{-1}\left(\frac{1}{U(1,1)}\right)$

By obtaining the phase delay caused by dielectric- graphene photonic crystal, the interference intensity of the two beams can be calculated.

\section{Gaussian Beam}

The output of He-Ne laser can be considered as a Gaussian beam. The field of Gaussian beam in the cylindrical coordinates is as follows [23]:

$$
\begin{aligned}
E(x, y, z) & =E_{0} \frac{\omega_{0}}{\omega(z)} \exp \left[-\frac{r^{2}}{\omega^{2}(z)}\right] \times \\
\exp \left\{-i\left[k z-\tan ^{-1}\left(\frac{z}{z_{0}}\right)+\frac{k r^{2}}{2 R(z)}\right]\right. &
\end{aligned}
$$

where $\omega_{0}$ is the spot size on the waist, $k$ is the propagation constant, and $z_{0}$ is the Rayleigh length. $\omega(z)$ and $R(z)$ are the spot size and the radius of curvature at the distance $z$ from the waist, respectively which are related through the following relationship:

$\frac{1}{q(z)}=\frac{1}{R(z)}-i \frac{\lambda}{\pi n \omega^{2}(z)}$

So if we have $q_{1}$ and consequently $R(z)$ and $\omega(z)$ at one point of the system, we can obtain the spot size and the radius of curvature and therefore the field of Gaussian beam at any desired point of the system by ABCD law:

$\frac{1}{q_{2}}=\frac{C+\frac{D}{q_{1}}}{A+\frac{B}{q_{1}}}$

where $A, B, C$ and $D$ are the transfer matrix elements of the system.

\section{III.SIMULATION RESULTS}

In this section, the effect of inserting dielectric-graphene photonic crystal on interference pattern and sensitivity of the ring laser gyroscope is simulated. Here we add the crystal in one arm of the RLG as shown in Fig. 3. In all simulations, the number of periods in the photonic crystal is 2 and the dielectric layer is silicon with a refractive index of 4.2060. 


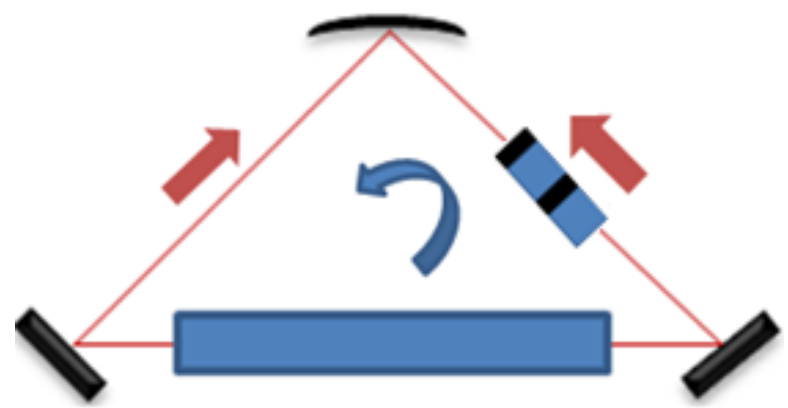

Fig. 3. A ring laser gyroscope with dielectricgraphene photonic crystal. The light is generated by the laser and transmitted through two outputs of the laser in two opposite directions.

As mentioned before, graphene has high optical transparency at visible wavelengths. In Fig. 4, the transmittance of dielectric-graphene photonic crystal is plotted as a function of wavelength ranged from $560 \mathrm{~nm}$ to $700 \mathrm{~nm}$. As shown in this figure, the dielectric-graphene photonic crystal has a high transmission at wavelength $632.8 \mathrm{~nm}$ and therefore by using this structure in the ring laser gyroscope doesn't decrease the efficiency of the device.

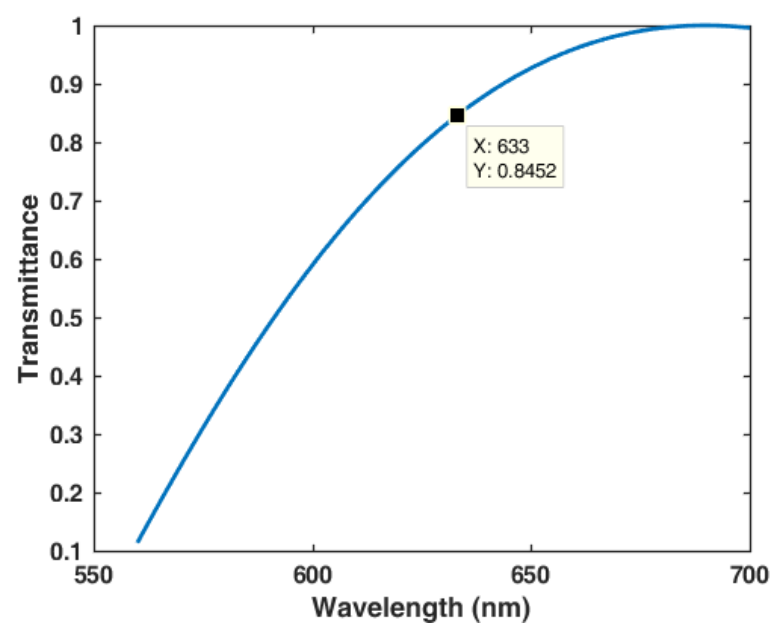

Fig. 4. The transmittance spectrum of dielectricgraphene photonic crystal.

The sensitivity is calculated from the following equation [24]:

$S=\frac{1}{I_{0}} \frac{d I_{i}}{d \varsigma}$

where $I_{0}=\frac{1}{2}\left|E_{0}\right|^{2}$ and $E_{0}$ is the amplitude of input field and $s$ is the parameter in which the sensitivity is measured in term of it. Figure 5 shows the comparison between a RLG with and without dielectric-graphene photonic crystal in terms of RLG arm length ranged from $0.2 \mathrm{~m}$ to $0.3 \mathrm{~m}$. As shown in this figure, when the dielectric-graphene photonic crystal is in the system, it is possible to build gyroscope with smaller lengths. Also, the sensitivity values of dielectric-graphene photonic crystal RLG are more than the case when there is no PC in the system which means that the phase difference between two interference beams is increased and leads to a more accurate measurement of the angular velocity of the system.

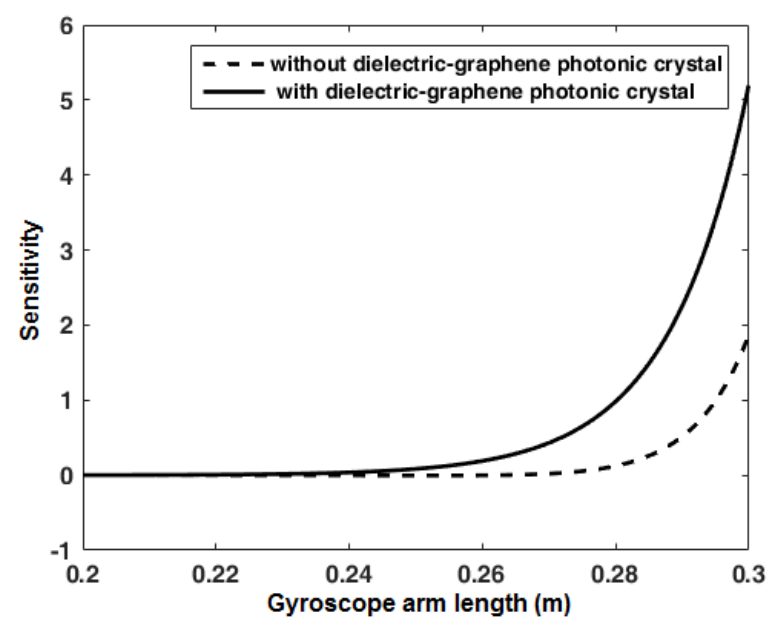

Fig. 5. Comparison of a gyroscope with and without dielectric-graphene photonic crystal vs. RLG arm length.

In Fig. 6, the interference intensity of the two beams $\left(I_{i}\right)$ is plotted in terms of distance to the central fringe on the screen $\left(Y_{m}\right)$ for three different cases. The dashed line corresponds to the case when there is no dielectric-graphene photonic crystal in the RLG, the solid line corresponds to the case when the thickness of dielectric layer is $d=0.09 \mu m$ and the dotted line corresponds to the case when the thickness of dielectric layer is $d=0.1 \mu \mathrm{m}$. As seen in this figure, as the thickness of the dielectric layer increases, the phase difference between the two beams increases compared to the case when there is no dielectric-graphene photonic crystal in the system.

In Fig. 7, the sensitivity coefficient $S$ is plotted versus the thickness of the dielectric layer. As 
shown, by increasing the thickness of the dielectric layer, the sensitivity of the device increases.

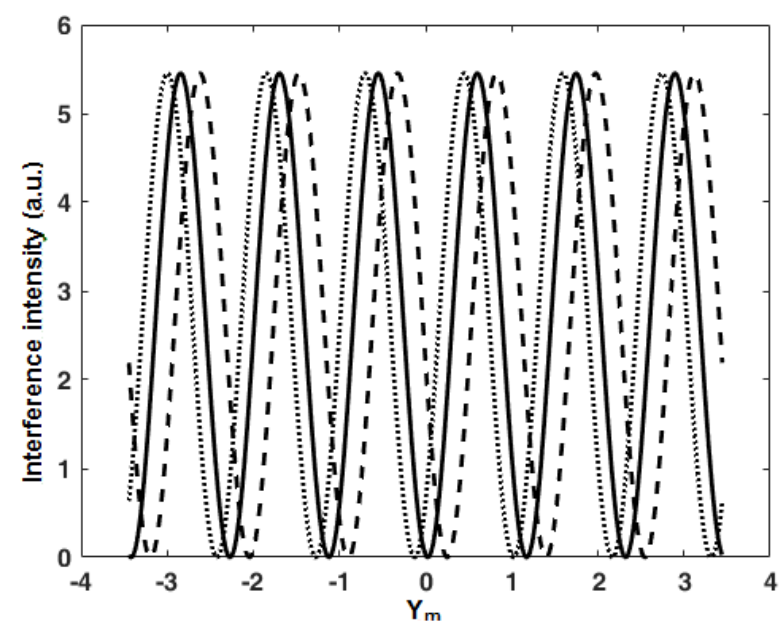

Fig. 6. Interference intensity vs. the distance to the central fringes (optional unit).

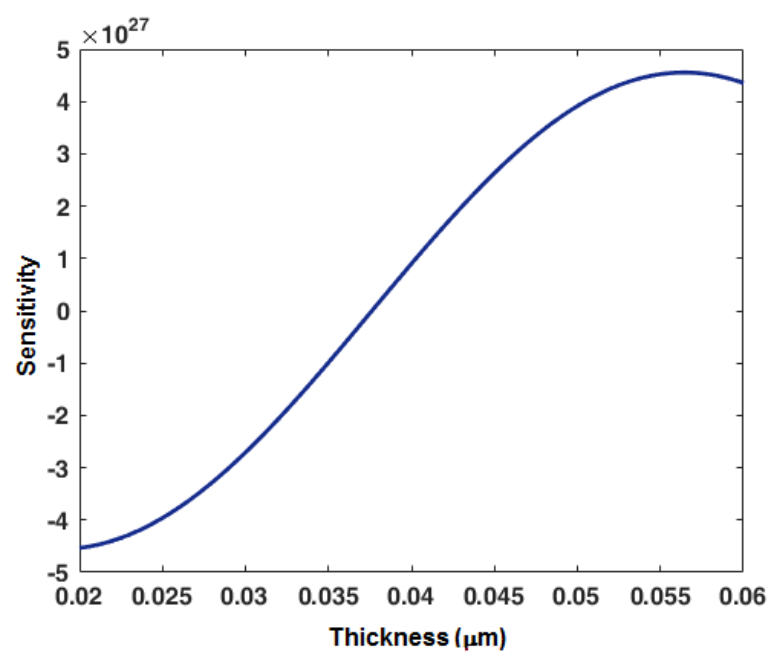

Fig 7. Sensitivity coefficient vs. the thickness of the dielectric layer.

\section{IV.CONCLUSION}

In this article, the ring laser gyroscope and its operation were first introduced. In RLGs the higher the phase difference, the better the interference fringes are detected and the sensitivity of the device goes up. Then the effect of inserting a dielectric-graphene photonic crystal inside a ring laser gyroscope on the interference pattern and the sensitivity of the device are studied and simulated using ABCD propagation matrix method. The results show that dielectric-graphene photonic crystal is a suitable choice to use in the RLG because it has a transmission of $80 \%$ in the wavelength of RLG. Also, a comparison between ring laser gyroscope with and without dielectricgraphene photonic crystal shows that when the dielectric-graphene photonic crystal is in the system it is possible to reduce the RLG size and it increases the phase difference between the two beams and therefore the sensitivity of the device increases.

\section{REFERENCES}

[1] V. Passaro, A. Cuccovillo, L. Vaiani, M. De Carlo, and C. E. Campanella, "Gyroscope Technology and Applications: A Review in the Industrial Perspective," Sensors, Vol. 17, pp. 2284 (1-22), 2017.

[2] P.R. Ayswarya, S.S. Pournami, and R. Nambiar, "A survey on ring laser gyroscope technology," Int. J. Comput. Appl., Vol. 116, pp. 25-27, 2015.

[3] J.E. Schaar, H. Yum, and S.M. Shahriar, "Theoretical description and design of a fast-light enhanced helium-neon ring-laser gyroscope," in Proc. SPIE, Vol. 7949, pp. 794914 (1-9), 2011.

[4] X. Han, H. Luo, T. Qu, Z. Wang, J. Yuan, and B. Zhang, "Theoretical design of a superluminal helium-neon ring laser via coupled passive cavities," J. Opt. Vol. 16, pp. 125401 (1-9), 2014.

[5] T. Qu, K. Yang, X. Han, S. Wu, Y. Huang, and H. Luo, "Design of a superluminal ring laser gyroscope using multilayer optical coatings with huge group delay,” Sci. Rep. Vol. 4, pp. 7098 (15), 2014.

[6] M. Salit, K. Salit, and P. E. Bauhahn, "Increasing the scale factor of a ring laser gyro via spectral hole burning," in Proc. SPIE, Vol. 8273, pp. 82730H (1-9), 2012.

[7] M.S. Shahriar, G.S. Pati, R. Tripathi, V. Gopal, M. Messall, and K. Salit, "Ultrahigh enhancement in absolute and relative rotation sensing using fast and slow light," Phys. Rev. A, Vol. 75, pp. 53807 (1-10), 2007.

[8] M. A. Terrel, M. J. F. Digonnet, and S. Fan, "Coupled resonator gyroscopes: what works and what does not," in Advances in Slow and Fast Light III, Vol. 7612, pp. 76120B (1-9), 2010.

[9] C. Fenzl, T. Hirsch, and O.S. Wolfbeis, "Photonic crystals for chemical sensing and biosensing," Angew. Chemie Int. Ed. Vol. 53, pp. 3318-3335, 2014.

[10] J.D. Joannopoulos, S.G. Johnson, J.N. Winn, and R.D. Meade, Photonic crystals: molding the flow of light, in optics \& light, $2^{\text {nd }}$ Ed. I. Gnerlich, Ed. New Jersey: Princeton university press, 2011. 
[11] K. Inoue and K. Ohtaka, Photonic crystals: physics, fabrication and applications, Springer, 2013.

[12] Z. Cai, N.L. Smith, J.-T. Zhang, and S.A. Asher, "Two-dimensional photonic crystal chemical and biomolecular sensors," Anal. Chem. Vol. 87, pp. 5013-5025, 2015.

[13] K. Ahmed and M. Morshed, "Design and numerical analysis of microstructured-core octagonal photonic crystal fiber for sensing applications," Sens. Bio-Sensing Res. Vol. 7, pp. $1-6,2016$.

[14] H. Wang and K.-Q. Zhang, "Photonic crystal structures with tunable structure color as colorimetric sensors," Sensors, Vol. 13, pp. 41924213, 2013

[15] Y. Yu, M. Heuck, H. Hu, W. Xue, C. Peucheret, Y. Chen, L.K. Oxenløwe, K. Yvind, and J. Mørk, "Fano resonance control in a photonic crystal structure and its application to ultrafast switching," Appl. Phys. Lett. Vol. 105, pp. 61117 (1-15) 2014.

[16] M. I. Hasan, M. S. Habib, M. S. Habib, and S. M. A. Razzak, "Highly nonlinear and highly birefringent dispersion compensating photonic crystal fiber," Opt. Fiber Technol. Vol. 20, pp. 32 $38,2014$.

[17] A. Goban, C.-L. Hung, S.-P. Yu, J.D. Hood, J.A. Muniz, J.H. Lee, M.J. Martin, A.C. McClung, K.S. Choi, and D.E. Chang, "Atom-light interactions in photonic crystals," Nat. Commun. Vol. 5, pp. 1-9, 2014.

[18] N. Segal, S. Keren-Zur, N. Hendler, and T. Ellenbogen, "Controlling light with metamaterialbased nonlinear photonic crystals," Nat. Photonics, Vol. 9, pp. 180-184, 2015.

[19] F. Bonaccorso, Z. Sun, T. Hasan, and A.C. Ferrari, "Graphene photonics and optoelectronics," Nat. Photonics, Vol. 4, pp. 611-622, 2010.

[20] M. Pizzocaro, "Development of a ring laser gyro: active stabilization and sensitivity analysis," $\mathrm{PhD}$ thesis, Dept. physics. Eng., Pisa Univ., Pisa, Italy, 2009.

[21] B. Wang, X. Zhang, K.P. Loh, J. and Teng, "Tunable broadband transmission and phase modulation of light through graphene multilayers", Appl. Phys. Vol. 115, pp. 213102 (1-8), 2014.

[22] S. L. Chuang, Physics of photonic devices, in Lasers \& Photonics, $2^{\text {nd }}$ Ed. G. Boreman, Ed. New Jersey, Wiley \& Sons, 2012.

[23] J.T. Verdeyen, Laser electronics, in lasers, $3^{\text {rd }}$ Ed. I. Zucker, Ed. New Jersey: Prentice Hall,1989.
[24] H. Wen, G. Skolianos, S. Fan, M. Bernier, R. Vallée, and M.J.F. Digonnet, "Slow-light fiberBragg-grating strain sensor with a 280femtostrain $/ \sqrt{ } \mathrm{Hz}$ resolution," J. Light. Technol. Vol. 31, pp. 1804-1808, 2013.

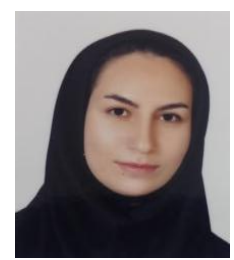

Maryam Soltani was born in Iran in 1992. She received her BS in optic and laser from Bonab University in 2015. She started her MS degree in 2015 in photonics at Shiraz University of Technology. Her master thesis was focused on ring laser gyroscope with the subject of "Investigation of the Effect of Inserting Photonic Crystal on Output of a Ring Laser Gyroscope". She is the author of 3 conference papers. She is a member of optics and photonics society of Iran.

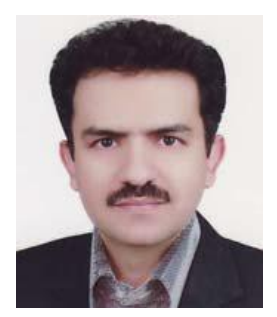

Alireza Keshavarz was born in Shiraz, Iran. He received his B.S. degree in Atomic Physics from Shiraz University in 1995, M.S. degree in Atomic and molecular Physics from Kerman University in 1997, and the Ph.D. degrees in Physics (laser and optics) from Shiraz University in 2004. He worked on much scientific research area such as Nonlinear Optics and Laser, Optical Solitons, NanoPhotonics and Nano-Physics (Quantum Dots and Wires). He joined the Department of physics of Shiraz University of Technology in 2004. He became Associate Professor in 2012 and Professor in 2016. At the present, he is actively involved in optics and photonics and has many M.S. and Ph.D. students in this field. 


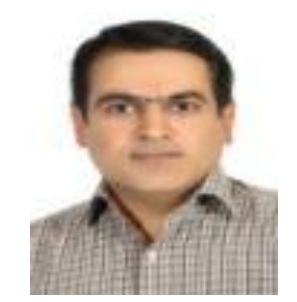

Gholamreza Honarasa was born in Iran at 1977. He received his Ph.D. from Yazd University with the title "The study of some nonclassical features of solvable quantum systems and quantum solitons in nonlinear medium" in 2012. He is now Assistant Professor at Shiraz University of Technology. He has published more than 16 papers and 1 book. His current research interests include laser, wave propagation and quantum optics. He is a member of physics society of Iran and optics and photonics society of Iran.

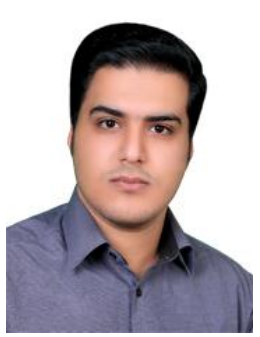

Reza Ghayoor was born in Isfahan, Iran. He received his B.S. degree in Optics and laser engineering from Maleke Ashtar University of Technology in 2015, M.S. degree in Photonic from Shiraz University of Technology in 2018. His master thesis was focused on solar cells with the subject of "Fabrication and Characterization of Dye Sensitized Solar Cell Containing Reduced Graphene Oxides". He is the author of more than 5 papers. He worked on scientific research area such as Dye sensitized solar cells, Terahertz waves, NanoPhotonics and Nano-Physics (Graphene and Quantum Dots). At the present, he is actively involved in optics and photonics. 
THIS PAGE IS INTENTIONALLY LEFT BLANK. 